\title{
Efeito da adição inulina sobre as características reológicas e térmicas da farinha de trigo
}

\author{
BARROS, J. H. T., ${ }^{1}$ FRANCO, C. M. L. ${ }^{1}$ \\ ${ }^{1}$ Universidade Estadual Paulista, Departamento de Engenharia e Tecnologia de Alimentos \\ E-mail: jefferson.ht.barros@hotmail.com
}

\begin{abstract}
RESUMO - Aumentar o teor de fibras em pães é um importante desafio da indústria de panificação. Neste estudo, o efeito da inulina de média polimerização (IMP) sobre as características reológicas e térmicas da farinha de trigo foi avaliado. Propriedades farinográficas, extensográficas, viscográficas e térmicas da farinha de trigo adicionada de $0,5,7,5$ e $10 \%$ de IMP foram analisadas. Pães elaborados com e sem IMP foram produzidos e avaliados quanto ao volume específico, cor e firmeza. Adição de IMP diminuiu a absorção de água da farinha, aumentou tempo de desenvolvimento e estabilidade da massa. A extensibilidade da massa diminuiu, enquanto a elasticidade aumentou gradativamente atingindo 1000UB com 7,5\% de IMP. O aumento da concentração de IMP diminuiu a entalpia de gelatinização e alterou as viscosidades de pasta da farinha. Os resultados mostraram que a adição de IMP provocou fortalecimento da farinha e que a concentração de 7,5\% foi a que menos interferiu na qualidade do pão, proporcionando volume, cor e textura semelhantes aos do pão controle.
\end{abstract}

\section{INTRODUÇÃO}

O mercado de pães vem crescendo rapidamente, cerca de $24 \%$ nos últimos cinco anos (Pegn, 2014) e esse crescimento demanda a criação de novos equipamentos, formulações, aditivos e ingredientes alimentícios, especialmente aqueles com propriedades funcionais (Morris e Morris). Com isso, o enriquecimento de pães com ingredientes funcionais é de interesse para o consumidor, bem como para a indústria de cereais. Estudos têm investigado o efeito da adição de diferentes fontes de fibras no pão (Hager et al., 2010) e das quais a inulina têm se destacado (Meyer e Peters, 2009; Morris e Morris, 2012).

A inulina é constituída de uma mistura de polímeros e oligômeros lineares de frutose (Leite et al., 2004). Sua ingestão resulta em muitos benefícios à saúde, como a modulação do metabolismo lipídico, a redução do risco de doenças como câncer de cólon e osteoporose, além de aumento significativo dos benefícios das bifidobactérias (Roberfroid, 2007).

A inulina exibe propriedades viscoelásticas que dependem do polímero, da concentração e do grau de polimerização (GP) (Zimeri e Kokini, 2003). Em panificação, ela pode afetar a consistência da massa, contribuir para a elasticidade global da mesma e interagir com o glúten (Wang et al., 2002). Inulinas com diferentes GP são encontradas comercialmente. Dentre elas, IMP (GP $\geq 10)$ é menos deletéria à qualidade do pão (Peressini e Sensidoni, 2009). No entanto, a fibra pode sofrer ação da invertase presente na levedura, bem como degradação pelo calor (Meyer e Peters, 2009). Inulina de maiores polimerização ( $\mathrm{GP} \geq 10)$ tem maior resistência às condições de 
processo (Morris e Morris, 2012).

Estudos controversos sobre o efeito da inulina em panificação têm sido encontrados. Peressini e Sensidoni (2009) estudaram o efeito de inulinas com vários GP's nas propriedades reológicas de dois tipos farinha de trigo (fraca e forte) e na qualidade dos pães. Esses autores observaram que, independente do GP, a inulina fortaleceu ambas as farinhas. No entanto, os pães obtidos apresentaram menor volume e coloração mais escura que aqueles sem a presença da fibra. Dentre as inulinas estudadas, IMP foi a que menos afetou as características do pão. Por outro lado, Wang et al. (2002) e Hager et al. (2011) observaram redução na elasticidade da massa e pães com maiores volumes quando estudaram o efeito dessas fibras em pães.

O objetivo deste trabalho foi avaliar o efeito da IMP sobre as características reológicas e térmicas da farinha de trigo e seu impacto na qualidade do pão.

\section{MATERIAL E MÉTODOS}

\subsection{Material}

Inulina GR (GP $\geq 10)$ gentilmente cedida pela Beneo-Orafti (Bélgica) e farinha de trigo com características panificáveis foram utilizadas neste estudo.

\subsection{Métodos}

Caracterização reológica: As características farinográficas e extensográficas da farinha de trigo adicionada de $0,5,7,5$ e $10 \%$ de IMP (em relação à massa total da farinha) foram analisadas usando um farinógrafo (mod. 810101, Brandender, Alemanha) e um extensógrafo (mod. 860000, Brandender, Alemanha) de acordo com os métodos 54-21 e 54-10, respectivamente, dos Approved Methods of American Association of Cereal Chemists (AACC, 2000). As análises foram realizadas em triplicata.

Propriedades de pasta: As propriedades de pasta da farinha adicionada de 0, 5, 7,5 e 10\% de IMP (em relação à massa total da farinha) foram determinadas usando um Rápido Visco Analisador (mod. RVA-4, Newport Scientific, Austrália) segundo método 76-21 da AACC (2000). As análises foram realizadas em triplicata.

Análise Térmica: As propriedades térmicas da farinha adicionada de 0, 5, 7,5 e 10\% de IMP (em relação à massa total da farinha) foram analisadas usando um calorímetro exploratório diferencial (DSC) (mod. Pyris 1, Perkin Elmer, USA) de acordo com Oliveira et al. (2014) com modificações. IMP foi solubilizada em água deionizada sob agitação antes de ser misturada à farinha. Amostras de farinha foram pesadas em cadinhos de alumínio, misturadas com água destilada com e sem IMP na proporção (1:3) e seladas em prensa específica do DSC. Os cadinhos selados foram mantidos a temperatura ambiente por $12 \mathrm{~h}$ e aquecidas a uma razão de $10{ }^{\circ} \mathrm{C} / \mathrm{min}$ de $25{ }^{\circ} \mathrm{C}$ a $125{ }^{\circ} \mathrm{C}$. Após análise, as amostras foram armazenadas a $4{ }^{\circ} \mathrm{C}$ por 14 dias e novamente analisadas nas mesmas condições. As temperaturas de gelatinização (inicial, de pico e final) e a variação de entalpia foram determinadas utilizando-se o software Pyris 1 (Perkin Elmer, USA). As análises foram realizadas em triplicata. 
Produção dos Pães: A produção dos pães seguiu metodologia proposta por El-Dash (1978). A formulação constou de $100 \%$ de farinha, $1,75 \%$ de sal, $3 \%$ de açúcar, $2 \%$ fermento seco, $3 \%$ óleo vegetal de milho. A quantidade de água usada foi aquela determinada em farinógrafo quando $0,5,7,5$ e $10 \%$ de inulina $(\% \mathrm{~m} / \mathrm{m}$ em relação à massa total da farinha) foram usadas. A etapa de mistura foi realizada no farinógrafo e o tempo requerido para o desenvolvimento do glúten foi aquele determinado pelo farinógrafo. A massa foi deixada descansar por $15 \mathrm{~min}$ a $32{ }^{\circ} \mathrm{C}$ e $80 \%$ UR, dividida em porções de $100 \mathrm{~g}$, boleada e deixada descansar por outros 15 min antes de ser moldada. Após moldagem a massa foi fermentada por $90 \mathrm{~min}$ a $32{ }^{\circ} \mathrm{C}$ e $80 \%$ UR e assada em forno com controle de vapor a $150{ }^{\circ} \mathrm{C}$ por $15 \mathrm{~min}$. Após forneamento, os pães foram resfriados por $2 \mathrm{~h}$ a temperatura ambiente antes de serem analisados.

Avaliação tecnológica: $\mathrm{O}$ volume dos pães controle e adicionados de IMP foram determinados, em triplicata, por deslocamento de sementes de acordo com o método 10-05 da AACC (2000). O volume específico do pão (VE) foi calculado como a relação entre o volume e o peso do pão $\left(\mathrm{cm}^{3} \cdot \mathrm{g}^{-1}\right)$. O sistema HunterLab foi usado para determinar a cor do miolo e da crosta dos pães controle e adicionados de IMP. Os parâmetros L* $\mathrm{a}^{*} \mathrm{~b}^{*}$ foram determinados em triplicata usando um colorímetro Colorflex 45/0 (HunterLab, EUA) e o software universal versão 4.1 foi usado para processar os resultados. A firmeza do miolo dos pães controle e adicionados de IMP foi determinada usando um texturômetro (TA - XT2i, Stable Micro Systems, UK) de acordo com método 74-09 da AACC (2000). Amostras de miolo de pão (25 mm de espessura e $20 \mathrm{~mm}$ de diâmetro) foram obtidas usando um molde de metal. A firmeza foi definida como a força em (N) necessária para comprimir a amostra a $40 \%$ de sua altura original, usando uma sonda circular com $25 \mathrm{~mm}$ de diâmetro, a intervalos de $5 \mathrm{~s}$ entre as compressões. Seis replicatas de cada amostra foram analisadas.

\subsection{Análise Estatística}

O delineamento experimental adotado foi o inteiramente casualizado. Os dados foram avaliados usando o programa Statística 7.0, abrangendo a análise de variância (ANOVA) e comparação de médias pelo teste de Tukey $(\mathrm{p} \leq 0,05)$.

\section{RESULTADOS E DISCUSSÃO}

Propriedades reológicas da farinha de trigo: O farinógrafo avalia o comportamento da farinha durante o processo de mistura da massa, enquanto o extensógrafo avalia sua elasticidade e extensibilidade, permitindo classificar a farinha quanto a sua força e ao seu uso adequado (Pizinatto, 1997).

A Tabela 1 apresenta o efeito da IMP sobre as propriedades farinográficas e extensográficas da farinha. De acordo com a classificação de Pizinatto (1997), a farinha de trigo usada neste trabalho pode ser classificada como forte a muito forte. A adição de IMP diminuiu a capacidade de absorção de água (AA) da farinha. Essa redução foi indiretamente proporcional à concentração de IMP. O tempo de desenvolvimento da massa e a estabilidade aumentaram, enquanto não houve alteração do índice de tolerância à mistura (ITM) com a adição de IMP, com exceção da concentração de $10 \%$ de IMP. Resultados semelhantes foram encontrados por KaroliniSkaradzinska et al. (2007) e Meyer e Peters (2009). De acordo com Rosell et al. (2010) a inulina interage bem com a estrutura da massa e aumenta sua estabilidade. 
Tabela 1: Propriedades farinográficas e extensográficas (135 min) da farinha de trigo controle e adicionada de 5, 7,5 e 10\% de IMP

\begin{tabular}{ccccc|ccc}
\hline \multicolumn{9}{c}{ Farinógrafo } & \multicolumn{3}{c}{ Extensógrafo } \\
\hline Amostra & $\begin{array}{c}\mathrm{AA}^{*} \\
(\%)\end{array}$ & $\begin{array}{c}\text { TD* } \\
(\mathrm{min})\end{array}$ & $\begin{array}{c}\mathrm{E}^{*} \\
(\mathrm{~min})\end{array}$ & $\begin{array}{c}\mathrm{ITM}^{*} \\
\left(\mathrm{BU}^{* *}\right)\end{array}$ & $\begin{array}{c}\mathrm{R}^{*} \\
\left(\mathrm{BU}^{* *}\right)\end{array}$ & $\begin{array}{c}\text { Ex* } \\
(\mathrm{mm})\end{array}$ & $\begin{array}{c}\mathrm{D}^{*} \\
(\mathrm{R} 50 / \mathrm{EX})\end{array}$ \\
\hline Controle & 58 & $10,3 \mathrm{~b}$ & $17,2 \mathrm{~b}$ & $10,0 \mathrm{~b}$ & $630 \mathrm{~b}$ & $149,5 \mathrm{a}$ & $4,2 \mathrm{~b}$ \\
$\mathbf{5 \%}$ IMP & 52 & $11,3 \mathrm{~b}$ & $29,0 \mathrm{a}$ & $13,5 \mathrm{~b}$ & $960 \mathrm{a}$ & $123,8 \mathrm{~b}$ & $7,8 \mathrm{a}$ \\
$\mathbf{7 , 5 \%}$ IMP & 53 & $17,5 \mathrm{a}$ & $28,0 \mathrm{a}$ & $10,0 \mathrm{~b}$ & $1000 \mathrm{a}$ & $117,8 \mathrm{~b}$ & $8,5 \mathrm{a}$ \\
$\mathbf{1 0 \%}$ IMP & 55 & $16,3 \mathrm{a}$ & $21,3 \mathrm{~b}$ & $19,0 \mathrm{a}$ & $1000 \mathrm{a}$ & $116,8 \mathrm{~b}$ & $8,6 \mathrm{a}$
\end{tabular}

*Capacidade de absorção de água (AA), tempo de desenvolvimento (TD), estabilidade (E), índice de tolerância a mistura (ITM), a resistência à extensão $\left(\mathrm{R}_{50}\right)$, extensibilidade $(\mathrm{EX})$ e número proporcional $(\mathrm{D})$. ** $\mathrm{BU}$ - Unidades Brabender. Médias de três replicatas. Valores seguidos de letras iguais na mesma coluna não diferem significativamente pelo teste de Tukey ( $>>0.05)$.

A massa necessita de um equilíbrio entre sua elasticidade e extensibilidade para tolerar as diferentes etapas do processo de panificação como mistura, fermentação, modelagem e forneamento (Pizinatto, 1997). A adição de IMP aumentou a resistência da massa à extensão (elasticidade) e diminuiu a extensibilidade, aumentando muito a força da farinha. Segundo Perissini e Sensidoni (2009) e Rossell et al. (2010), as cadeias de inulina podem interagir entre si e com as proteínas do glúten formando redes elásticas e aumentando a elasticidade da massa.

O uso de fibras alimentares em panificação geralmente enfraquece a massa e assim o uso de farinhas fortes é recomendado para a fabricação de pães adicionados das mesmas (Morris e Morris, 2012). Porém, neste trabalho, a adição de IMP fortaleceu a massa. Esse efeito seria indicado especialmente no caso de farinhas de trigo de força mediana, conferindo maior força e melhorando a retenção de gás durante a fermentação.

Propriedades viscográficas da farinha de trigo: Quando a farinha é aquecida na presença de água, o amido sofre alterações irreversíveis que são dependentes da disponibilidade de água, da temperatura e da concentração de enzimas endógenas. (Thomas e Atwell, 1999). Durante o resfriamento da pasta ocorre a reorganização das moléculas de amido, fenômeno denominado de retrogradação (Atwell, 2001).

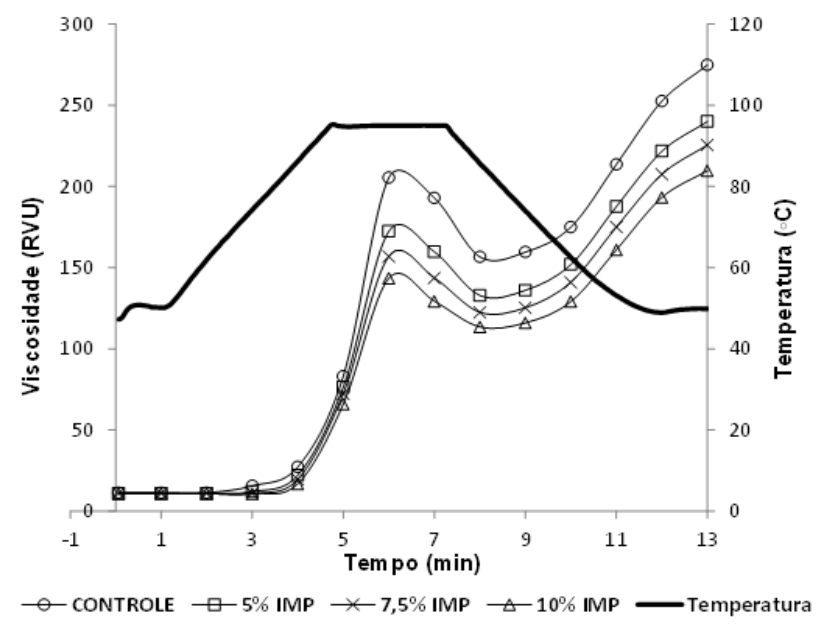

Figura 1: Perfil viscoamilográfico da farinha de trigo sem e com IMP. 
A adição de IMP alterou as propriedades de pasta da farinha (Figura 1). A temperatura de pasta não foi significativamente alterada, porém as viscosidades de pico, de quebra, final e setback diminuíram quanto maior a concentração de IMP. A inulina é altamente hidrofílica, e reduz a disponibilidade da água para inchamento dos grânulos de amido. $\mathrm{O}$ inchaço limitado do amido diminui o seu volume e, consequentemente, o atrito entre as moléculas, o qual se manifesta como menor viscosidade (Peressini e Sensidoni, 2009). Durante o aquecimento da suspensão de farinha, a presença de oligossacarídeos pode ter dificultado a lixiviação da amilose diminuindo o setback.

Propriedades térmicas da farinha de trigo: A farinha de trigo mostrou faixa de temperatura de gelatinização de 58,77 a $69,44{ }^{\circ} \mathrm{C}$ com uma variação de entalpia de gelatinização de $8,15 \mathrm{~J} / \mathrm{g}$ (Tabela 2). Puppo et al. (2005) encontraram resultados semelhantes em farinhas de trigo com diferentes quantidades de proteína.

Tabela 2: Propriedades de gelatinização e retrogradação da farinha de trigo sem e com IMP

\begin{tabular}{ccccc|ccccc}
\hline \multicolumn{5}{c|}{ Gelatinização } & \multicolumn{5}{c}{ Retrogradação } \\
\hline Amostra & To & Tp & Tf & $\Delta \mathrm{Hg}$ & To & Tp & Tf & $\Delta \mathrm{Hg}$ & $\% \mathrm{RT}$ \\
& $\left({ }^{\circ} \mathrm{C}\right)$ & $\left({ }^{\circ} \mathrm{C}\right)$ & $\left({ }^{\circ} \mathrm{C}\right)$ & $(\mathrm{J} / \mathrm{g})$ & $\left({ }^{\circ} \mathrm{C}\right)$ & $\left({ }^{\circ} \mathrm{C}\right)$ & $\left({ }^{\circ} \mathrm{C}\right)$ & $(\mathrm{J} / \mathrm{g})$ & \\
\hline Controle & $58,77 \mathrm{a}$ & $64,28 \mathrm{a}$ & $69,44 \mathrm{a}$ & $8,15 \mathrm{a}$ & $45,43 \mathrm{~b}$ & $55,04 \mathrm{~b}$ & $63,38 \mathrm{~b}$ & $0,73 \mathrm{~d}$ & $8,96 \mathrm{c}$ \\
$\mathbf{5 \% ~ I M P}$ & $58,21 \mathrm{~b}$ & $63,18 \mathrm{a}$ & $69,47 \mathrm{a}$ & $7,72 \mathrm{~b}$ & $50,37 \mathrm{a}$ & $59,82 \mathrm{a}$ & $67,40 \mathrm{a}$ & $1,24 \mathrm{a}$ & $16,06 \mathrm{a}$ \\
$\mathbf{7 , 5 \%} \mathbf{I M P}$ & $58,41 \mathrm{ab}$ & $64,10 \mathrm{a}$ & $69,38 \mathrm{a}$ & $7,72 \mathrm{~b}$ & $50,88 \mathrm{a}$ & $59,03 \mathrm{a}$ & $66,64 \mathrm{a}$ & $1,04 \mathrm{c}$ & $13,47 \mathrm{~b}$ \\
$\mathbf{1 0 \%}$ IMP & $58,56 \mathrm{ab}$ & $64,21 \mathrm{a}$ & $69,80 \mathrm{a}$ & $7,98 \mathrm{ab}$ & $51,40 \mathrm{a}$ & $55,04 \mathrm{a}$ & $65,68 \mathrm{ab}$ & $1,11 \mathrm{~b}$ & $13,90 \mathrm{~b}$ \\
\hline
\end{tabular}

Média de três replicatas. Valores seguidos pela mesma letra na mesma coluna não diferem significativamente pelo teste de Tukey $(\mathrm{p}>0,05) . \mathrm{T}_{0}, \mathrm{Tp}, \mathrm{Tf}=$ temperaturas inicial, de pico e final, respectivamente; $\Delta \mathrm{Hg}=$ entalpia de gelatinização; $\Delta \mathrm{Hr}=$ entalpia de retrogradação. $\mathrm{TR}=$ Taxa de retrogradação $(\Delta \mathrm{Hret} / \Delta \mathrm{Hg} * 100)$.

A presença de IMP em qualquer concentração não alterou as propriedades térmicas de gelatinização do amido presente na farinha, com exceção da amostra adicionada de 5\% de IMP que sofreu leve redução de sua temperatura inicial de gelatinização e variação de entalpia. Esses resultados corroboram com aqueles observados para as propriedades de pasta da farinha adicionadas de IMP. Apesar da fibra competir com o amido pela água, nas condições deste experimento, houve, independente da concentração de fibras, água suficiente para promover a gelatinização do amido, indicando que não houve interação da IMP com o amido.

Com o resfriamento, as moléculas do amido, principalmente a amilopectina se reorganizam novamente formando uma estrutura mais organizada (Atwell, 2001; Singh, 2010). O pico endotérmico de retrogradação da farinha sem IMP foi observado na faixa de 45,43 a $63,38{ }^{\circ} \mathrm{C}$. A presença de IMP provocou aumento nas temperaturas e entalpia de retrogradação sugerindo que a presença de IMP induz à formação de uma estrutura mais organizada durante a retrogradação do amido. No entanto, a farinha adicionada de 7,5\% de IMP mostrou a menor taxa de retrogradação.

Propriedades tecnológicas dos pães sem e com IMP: O volume final do pão, assim como a maciez e a cor da crosta são características importantes a serem consideradas na aceitação dos pães pelos consumidores (Tabela 3). 
Tabela 3: Volume específico, firmeza e cor do miolo e crosta dos pães* sem e com IMP

\begin{tabular}{ccc|ccc|ccc}
\hline Amostra & $\begin{array}{c}\text { VE } \\
\left(\mathrm{cm}^{3} / \mathrm{g}\right)\end{array}$ & Firmeza & \multicolumn{3}{|c|}{ Miolo } & \multicolumn{3}{|c}{ Crosta } \\
& $\mathrm{N})$ & $\mathrm{L}^{*}$ & $\mathrm{a}^{*}$ & $\mathrm{~b}^{*}$ & $\mathrm{~L}^{*}$ & $\mathrm{a}^{*}$ & $\mathrm{~b}^{*}$ \\
\hline Controle & $5,12 \mathrm{a}$ & $0,94 \mathrm{~d}$ & $76,16 \mathrm{a}$ & $0,91 \mathrm{~b}$ & $18,79 \mathrm{a}$ & $58,85 \mathrm{a}$ & $14,41 \mathrm{~b}$ & $36,03 \mathrm{a}$ \\
$\mathbf{5 \%}$ IMP & $4,70 \mathrm{ab}$ & $1,51 \mathrm{~b}$ & $73,40 \mathrm{~b}$ & $0,45 \mathrm{~d}$ & $18,30 \mathrm{a}$ & $52,54 \mathrm{~b}$ & $17,03 \mathrm{a}$ & $35,21 \mathrm{a}$ \\
$\mathbf{7 , 5 \%}$ IMP & $4,81 \mathrm{ab}$ & $1,26 \mathrm{c}$ & $75,63 \mathrm{ab}$ & $0,82 \mathrm{c}$ & $19,03 \mathrm{a}$ & $53,21 \mathrm{~b}$ & $16,79 \mathrm{a}$ & $35,00 \mathrm{a}$ \\
$\mathbf{1 0 \%}$ IMP & $4,62 \mathrm{~b}$ & $1,87 \mathrm{a}$ & $75,75 \mathrm{ab}$ & $0,97 \mathrm{a}$ & $19,05 \mathrm{a}$ & $49,56 \mathrm{~b}$ & $16,19 \mathrm{a}$ & $32,39 \mathrm{~b}$ \\
\hline
\end{tabular}

*Média de nove, seis e três replicatas. Respectivamente.Valores seguidos pela mesma letra na mesma coluna não diferem significativamente pelo teste de Tukey $(\mathrm{p}>0,05)$.

A adição de IMP provocou redução do VE e aumento da firmeza do miolo, devido ao fato da IMP ter aumentado a elasticidade da farinha de trigo, conferindo assim maior resistência à massa durante a fermentação.

A adição de IMP reduziu levemente o volume específico dos pães, sendo este efeito observado significativamente apenas na concentração de 10\% de IMP (Tabela 3). Esses resultados podem ter sido resultado da maior elasticidade observada para as massas adicionadas de IMP (Tabela 2). As propriedades da estrutura do glúten durante a fermentação e cozimento influenciam as propriedades do produto final, pois o glúten deve ter extensibilidade suficiente para expandir sobre influência do $\mathrm{CO}_{2}$ formado durante a fermentação, contribuindo para o volume do pão, mas também fornecendo o grau ótimo de resistência à extensão para poder reter sua forma (El Dash, 1982). A resistência à extensão muito alta resulta em um baixo volume, pois confere rigidez à massa, tornando-a incapaz de expandir a um volume ótimo. Meyers e Peters (2009) também observaram redução no volume do pão quando usaram inulinas de diferentes GP's. Esses autores observaram que a inulina de alta polimerização ( $\mathrm{GP} \geq 23$ ), mostrou efeito mais deletério ao volume do pão. Filipovic et al. (2010) também observaram menores volumes para pães adicionados de 5\% de IMP, enquanto que a adição de inulina de baixa polimerização resultou em pães com volumes maiores que o controle.

As concentrações de IMP aumentaram a firmeza do miolo dos pães, sendo esta menos evidenciada na concentração de 7,5\% de IMP. Este resultado corrobora com aquele observado para as propriedades térmicas da farinha que mostrou que entre as amostras com IMP, aquela com $7,5 \%$ de fibra mostrou a menor taxa de retrogradação (Tabela 3). Wang et al. (2002) e Hager et al. (2011) também observaram aumento na firmeza dos pães quando inulina foi adicionada. O aumento da firmeza do miolo pode ser atribuída a diferenças nas propriedades elásticas da massa e a redução da capacidade de retenção de gás devido à interação das fibras com a rede de glúten resultando num menor volume de pão (Peressini e Sensidoni, 2009).

A adição de IMP praticamente não alterou a luminosidade do miolo, mas os resultados mostraram que os pães com maiores VE resultaram em miolo com maior $\mathrm{L}^{*}$, pois pães com maior volume têm as células do miolo mais uniformes e, portanto refletem melhor a luz proporcionando maior luminosidade.

A L* das crostas variou de 58,85 a 49,86, sendo inversamente proporcional à concentração de IMP adicionada à farinha. $\mathrm{O}$ aumento da cor da crosta é resultado da reação não-enzimática entre os açúcares redutores e os grupos amino primários (Reação de Maillard) durante o forneamento. Não houve diferença na cor da crosta dos pães com diferentes concentrações de inulina. 


\section{CONCLUSÃO}

A adição de IMP provoca aumento na estabilidade e elasticidade da farinha, aumentando sua força, o que pode conferir menor capacidade de retenção de gás da massa e com isso resultar em pães com menor volume específico e maior firmeza, além da IMP potencializar a Reação de Maillard conferindo aos pães, crosta de coloração mais escura. No entanto, nas concentrações utilizadas neste trabalho, a adição de 7,5 \% de IMP propiciou pães de boa qualidade com volume, firmeza e cor próximos àqueles do pão controle.

\section{REFERÊNCIAS}

AACC, American association of cereal chemistry. Approved methods of the AACC. 10th. ed. Washington: St. Paul, Minn.: The Committee, 2000.

ATWELL, W. A. Wheat flour: practical guide for the food industry. St. Paul: American association of cereal chemists, 134p. 2001.

EL-DASH, A. A., CAMARGO, C. O.; DIAZ, N. M. Fundamentos da Tecnologia de Panificação. 345 p., 1982.

EL-DASH, A. A. Standardized mixing and fermentation procedure for experimental baking test. Cereal Chemistry, v. 55, p. 336-446, 1978.

FILIPOVIC, J.; FILIPOVIC, N.; FILIPOVIC, V. The effects of commercial fibres on frozen bread dough, Journal of the Serbian Chemical Society, vol. 75, p. 195-207, 2010.

HAGER, A. S.; RYAN, A.M.; SHWAB, C.; GANGLE, M. G.; O’DOHERTY, J. V.; ARENDT, E. E. Influence of the soluble fibres inulin and oat $\beta$-glucan on quality of dough and bread. Eur. Food Res Technol, v. 232, n. 1, p. 405-413, Dec. 2010.

KAROLINI-SKARADZINSKA, Z.; BIHUNIAK, P.; PIOTROWSKA, E.; WDOWIK, L. Properties of dough and qualitative characteristics of wheat bread with addition of inulin. Polish Journal of Food and Nutrition Sciences, vol. 57, p. 267-270, 2007.

LEITE, J.T.C.; PARK, K.J.; RAMALHO, J.R.P.; FURLAN, D.M. Caracterização reológica das diferentes fases de extrato de inulina de raízes de chicória, obtidas por abaixamento de temperatura. Engenharia Agrícola, v.24, p.202-210, 2004.

MEYER, D.; PETERS, B. Enhancing the nutritional value of bread with inulin. Agro Food Industry Hi-Tech, v. 20, p.48-50, 2009.

MORRIS, C.; MORRIS, G. A. The effect of inulin and fructo-oligosaccharide supplementation on the textural, rheological and sensory properties of bread and their role in weight management: A review. Food Chemistry, v. 133, p. 237-248, 2012.

OLIVEIRA, D. S.; TELIS-ROMERO, J.; DA-SILVA, R.; FRANCO, C. M. L. Effect of a Thermoascus aurantiacus thermostable enzyme cocktail on wheat bread qualitiy. Food Chemistry, v. 143, p. 139-146, 2014. 
PEGN. Revista pequenas empresas grandes negócios: Setor de pães industrializados fatura R 3,5 bi em 2012. Disponível em: <http://revistapegn.globo.com/Revista/Common/0,EMI33436217180,00.html>. Acesso em: 14 Abr. 2014

PERESSINI, D.; SENSIDONI, A. Effect of soluble dietary fibre addition on rheological and breadmaking properties of wheat doughs. Journal of Cereal Science, v. 49, p. 190-201, 2009.

PIZZINATTO, A. Qualidade da farinha de trigo: conceitos, fatores determinantes, parâmetros de avaliação e controle. Campinas: ITAL, 162p, 1997.

PUPPO, M. C; CALVELO, A; AÑóN, M. C. Physicochemical and rheological characterization of wheat flour. Cereal Chemestry, v. 82, p. 173-181, 2005

ROBERFROID, M. B. Inulin-Type Fructans: Functional Food Ingredients. Journal of Nutrition, v. 137, p.2493-2502, 2007.

ROSELL, C. M.; SANTOS, E.; COLLAR, C. Physical characterization of fiber enriched bread doughs by dual mixing and temperature constraint using the Mixolab(A (R)). European Food Research and Technology, vol. 231, p. 535-544, 2010.

SINGH, S.; SINGH, N.; ISONO, N.; NODA, T. Relationship of Granule Size Distribution and amylopectin structure with pasting, thermal, and retrogradation properties in wheat starch. Journal of Agricultural and Food Chemistry, v. 58, p. 1180-1188, 2010.

THOMAS, D. J; ATWEL, W. A. Starch. 1ed. St. Paul: Eagan Press, 1999.

WANG, J. S.; ROSELL, C. M.; DE BARBER, C. B. Effect of the addition of different fibres on wheat dough performance and bread quality. Food Chemistry, v. 79, p. 221-226, 2002.

ZIMERI, J.E.; KOKINI, J.L. Rheological properties of inulin-waxy maize starch systems. Carbohydrate Polymers, v. 52, p. 67-85, 2003.

\section{AGRADECIMENTOS}

Os autores agradecem a FAPESP e ao CNPq pelo suporte financeiro. 